\title{
GENETIC INVESTIGATION OF SNAKE RIVER AND YELLOWSTONE CUTTHROAT TROUT
}

\section{JEFFRY B. MITTON $\uparrow$ DEPARTMENT OF ECOLOGY AND EVOLUTIONARY BIOLOGY UNIVERSITY OF COLORADO $\uparrow$ BOULDER, CO}

\begin{abstract}
$\uparrow \quad$ SUMMARY
The relationship between Yellowstone cutthroat trout, Oncorhynchus clarki bouvieri, and Snake River finespotted cutthroat trout, $O$. clarki behnkei, was examined with two mitochondrial DNA fragments, COI and ND5. No variation was found within either subspecies, and just one (out of 1069) nucleotide differed between subspecies. Thus, these subspecies are very closely related.
\end{abstract}

Samples for this study were obtained from hatcheries, and may not be representative of the subspecies. O. c. bouveri were sampled from the Clark Fork Hatchery, in Powell, WY, and $O$. c. behnkei were sampled from the National Fish Hatchery in Jackson, WY. Further sampling, preferably from natural populations, is needed to more thoroughly survey the variation within subspecies, and to measure the differences between subspecies.

\section{$\uparrow \quad$ INTRODUCTION}

In the upper portion of the Snake River drainage, two subspecies of cutthroat trout can be distinguished by the sizes of their spots. The Snake River finespotted cutthroat, Oncorhynchus clarki behnkei, and Yellowstone cutthroat trout, O. clarki bouveri, both occupy the Snake River drainage between Jackson Lake and Palisades Reservoir, but Yellowstone occupies the smaller streams, while finespotted are in the main stem of the Snake River. Despite contact in several streams, the subspecies remain distinct for their spotting pattern. However, none of the meristic characters differ between these subspecies, and geneticists have not yet reported any diagnostic differences of any kind.

The purpose of this study was to survey these subspecies for diagnostic genetic markers. Mitochondrial sequences were chosen for their high rates of evolution and their maternal inheritance without recombination.

\section{MATERIALS AND METHODS}

Samples

Kerry Grande provided Snake River cutthroat from the National Fish Hatchery in Jackson, WY. Dave Miller provided Yellowstone cutthroat taken from the Lahar Rapids of the Yellowstone River, and currently in production at the Clark Fork Hatchery in Powell, WY. Arlene Ganek provided Rio Grande cutthroat, $O$. c. virginalis, from the Colorado Division of Wildlife Poudre River fish hatchery, which are a mixture of fish from different rivers. Brood fish from Mindano Creek, West Indian Creek and Placer Creek were all crossbred to create the hatchery stock at the Poudre River facility. The Rio Grande cutthroat in those three streams are considered to be "pure" Rio Grande cutthroat. Colorado Division of Wildlife personnel, including Douglas Krieger, Tom Nesler, and Jim Melby, provided samples of DNA from greenback cutthroat trout, $O$. $c$. 
stomias, from Severy Creek, Como Creek, and Graneros Creek. Biologists in the US Fish and Wildlife Service and Rocky Mountain National Park supplied Colorado River cutthroat, O. $c$. pleuriticus, from Ptarmigan Creek and Columbine Creek in Rocky Mountain National Park.

\section{Molecular methods}

Genomic DNA was extracted from a small piece of the adipose fin, using the manufacturer's instructions for the AIAamp Tissue Kit, marketed by Qiagen.

MtDNA sequences were obtained for COX I and ND5. The primers for COX I were COIared, (5' CACAGTGTGTAGGCGTCTGG 3') and COIf (5' CCTGCAGGAGGAGGAGACCC 3 '); both primers were modified from Palumbi (1996) to better fit cutthroat trout sequences. The primers for ND5 were ND5-2 (5' AATAGTTTAT CCGTTGGTCTTAGG 3') and ND5R1 (5' GGCC AAGATCTCCTACTCG 3'). Amplifications were conducted in a total volume of $50 \mu 1$ using 50 $\mathrm{mM}$ KCL, $10 \mathrm{mM}$ Tris-HCL (pH 8.3), $0.01 \%$ gelatin, $2 \mathrm{mM} \mathrm{MgCl} 2,200 \mu \mathrm{M}$ dNTPs, 1.5 units Taq polymerase, $0.3 \mu \mathrm{M}$ of each primer, $100 \mathrm{ng}$ template DNA and water to the final volume. PCR cycle conditions for COX I consisted of an initial denaturing step at $95^{\circ} \mathrm{C}$ for one minute followed by 39 cycles of 1 minute at $95^{\circ} \mathrm{C}, 1$ minute at $55^{\circ} \mathrm{C}$ and 3 minutes at $72^{\circ} \mathrm{C}$, capped off by a final step at $72^{\circ} \mathrm{C}$ for 7 minutes. The ND5 cycle conditions were modified from this program with an annealing temperature of $50^{\circ} \mathrm{C}$, an extension time of 1 minute, and 35 cycles.

All sequences were generated using Big Dye chemistry and an ABI377 in the sequencing facility in the Department of Molecular, Cellular, and Developmental Biology at the University of Colorado in Boulder. Sequences were edited, assembled into contigs, and arranged into correct reading frame with the software Sequencher.

The COX I and ND5 sequences were trimmed to $560 \mathrm{bp}$ and $509 \mathrm{bp}$, respectively. These were amplified, sequenced, edited and aligned separately, but the sequences for each fish were combined into a single 1069 bp sequence for comparison.

\section{RESULTS AND DISCUSSION}

Sequences were obtained for six Yellowstone and six finespotted cutthroat. No variation was found within either subspecies, but it is worth noting that each subspecies was sampled from a single hatchery. One diagnostic difference was noted between these subspecies, in the portion of the sequence from the COXI gene (position \#12, table 1).

For comparison to the subspecies occurring in further south, a sequence is presented that is shared by Colorado River, greenback, and Rio Grande cutthroat. The sequences in Yelllowstone and Snake River finespotted differ from this sequence by 9 and 10 substitutions, respectively (Table 1).

This study is the first to find a genetic difference between Yellowstone and Snake River finespotted cutthroat, but this result is offered tentatively. The Snake River cutthroat all came from one hatchery, and the Yellowstone all came from a different hatchery. It is likely that those hatchery stocks do not represent all of the variation within their subspecies. The stocks in those two hatcheries have different haplotypes, but does that indicate that the two subspecies are different? I recommend that each of these subspecies be sampled more thoroughly, from natural populations, if possible. This report also provides the incentive to survey further in the mitochondrial in the mitochondrial genome, focusing on the fastest-evolving genes, such as ND2.

\section{$\uparrow \quad$ Literature Cited}

Palumbi, S. R. 1996. Nucleic acids II: The polymerase chain reaction. pp. 205-247 in D. M. Hillis, C. Moritz, and B. K. Mable (eds.) Molecular Systematics: Second Edition. Sinauer Associates, Inc. Sunderland, MA. 
Table 1. Mitochondrial DNA sequences from cutthroat trout. The sequences are from COXI (first $560 \mathrm{bp}$ ) and ND5 (509 bp) in Yellowstone, Snake River finespotted, and three subspecies in Colorado; greenback, Colorado River, and Rio Grande cutthroat.

$\begin{array}{ll}\text { G CR RG } & \# 1 \\ \text { Snake River } & \# 1 \\ \text { Yellowstone } & \# 1 \\ & \\ & \end{array}$

G CR RG

Snake River

Yellowstone

G CR RG

Snake River

Yellowstone

G CR RG

Snake River

Yellowstone

G CR RG

Snake River

Yellowstone

G CR RG

Snake River

\#201

Yellowstone

\#201

\#201

\#201

G CR RG

Snake River

Yellowstone

\#241

\#241

\#241

G CR RG
GTCTATATTC TTATCCTCCC AGGCTTTGGT ATGATTTCAC GTCTATATTC TCATCCTCCC AGGCTTTGGT ATGATTTCAC GTCTATATTC TTATCCTCCC AGGCTTTGGT ATGATTTCAC

GTCTATATTC TTATCCTCCC AGGCTTTGGT ATGATTTCAC

ATATCGTTGC ATACTACTCC GGCAAAAAAG AACCCTTCGG ATATCGTTGC ATACTACTCC GGCAAAAAAG AACCCTTCGG ATATCGTTGC ATACTACTCC GGCAAAAAAG AACCCTTCGG

ATATATAGGA ATAGTCTGAG CTATAATAGC CATCGGATTA ATATATAGGA ATAGTCTGAG CTATGATAGC CATCGGATTA ATATATAGGA ATAGTCTGAG CTATGATAGC CATCGGATTA

ATATATAGGA ATAGTCTGAG CTATGATAGC CATCGGATTA

$*$

TTAGGATTTA TCGTTTGAGC CCACCATATG TTTACTGTCG TTAGGATTTA TCGTTTGAGC CCACCATATG TTTACTGTCG TTAGGATTTA TCGTTTGAGC CCACCATATG TTTACTGTCG

TTAGGATTTA TCGTTTGAGC CCACCATATG TTTACTGTCG

GGATAGACGT GGACACTCGT GCCTACTTTA CATCTGCCAC GGATAGACGT GGACACTCGT GCCTACTTTA CATCTGCCAC GGATAGACGT GGACACTCGT GCCTACTTTA CATCTGCCAC

GGATAGACGT GGACACTCGT GCCTACTTTA CATCTGCCAC

CATGATTATC GCTATCCCTA CAGGAGTAAA AGTATTTAGC CATGATTATC GCTATCCCTA CAGGAGTAAA AGTATTTAGT CATGATTATC GCTATCCCTA CAGGAGTAAA AGTATTTAGT

CATGATTATC GCTATCCCTA CAGGAGTAAA AGTATTTAGT

TGACTAGCCA CGCTACACGG AGGCTCGATC AAATGAGAAA TGACTAGCCA CACTACACGG AGGCTCGATC AAATGAGAAA TGACTAGCCA CACTACACGG AGGCTCGATC AAATGAGAAA

TGACTAGCCA CACTACACGG AGGCTCGATC AAATGAGAAA
$*$

CACCACTTCT TTGAGCCCTC GGGTTCATTT TCCTATTTAC 
Snake River Yellowstone

G CR RG

G CR RG

Snake River Yellowstone

G CR RG

Snake River Yellowstone

G CR RG Snake River
CACCACTTCT TTGAGCCCTC GGGTTCATTT TCCTATTTAC CACCACTTCT TTGAGCCCTC GGGTTCATTT TCCTATTTAC

CACCACTTCT TTGAGCCCTC GGGTTCATTT TCCTATTTAC

AGTGGGCGGA CTTACGGGTA TTGTCCTTGC TAACTCCTCA AGTGGGCGGA CTTACGGGTA TTGTCCTTGC TAACTCCTCA AGTGGGCGGA CTTACGGGTA TTGTCCTTGC TAACTCCTCA

AGTGGGCGGA CTTACGGGTA TTGTCCTTGC TAACTCCTCA

TTAGACATTG TTCTACATGA CACTTACTAC GTAGTTGCTC TTAGACATTG TTCTACATGA CACTTACTAC GTAGTTGCTC TTAGACATTG TTCTACATGA CACTTACTAC GTAGTTGCTC

TTAGACATTG TTCTACATGA CACTTACTAC GTAGTTGCTC

ATTTCCACTA CGTATTATCC ATAGGAGCTG TGTTTGCTAT ATTTCCACTA CGTATTATCT ATAGGAGCTG TGTTTGCTAT ATTTCCACTA CGTATTATCT ATAGGAGCTG TGTTTGCTAT

ATTTCCACTA CGTATTATCT ATAGGAGCTG TGTTTGCTAT *

TATAGGCGCT TTCGTACACT GATTTCCCCT ATTCACAGGA TATAGGCGCT TTCGTACACT GATTTCCCCT ATTTACAGGA TATAGGCGCT TTCGTACACT GATTTCCCCT ATTTACAGGA

TATAGGCGCT TTCGTACACT GATTTCCCCT ATTTACAGGA

TATACCCTTC ACAGCACATG GACCAAAATC CATTTTGGAA TACACCCTTC ACAGCACATG GACCAAAATC CATTTTGGAA TACACCCTTC ACAGCACATG GACCAAAATC CATTTTGGAA

TACACCCTTC ACAGCACATG GACCAAAATC CATTTTGGAA

TTATATTTAT CGGCGTAAAT TTAACCTTTT TCCCACAGCA TTATATTTAT CGGCGTAAAT TTAACCTTTT TCCCACAGCA TTATATTTAT CGGCGTAAAT TTAACCTTTT TCCCACAGCA

TTATATTTAT CGGCGTAAAT TTAACCTTTT TCCCACAGCA

TCCGACTACA CTCATCTTAA GCTCATCCCT TTTAACAATC TCCGACTACA CTCATCTTAA GCTCATCCCT TTTAACAATC TCCGACTACA CTCATCTTAA GCTCATCCCT TTTAACAATC
TCCGACTACA CTCATCTTAA GCTCATCCCT TTTAACAAT 
$\begin{array}{ll}\text { G CR RG } & \# 601 \\ \text { Snake River } & \# 601 \\ \text { Yellowstone } & \# 601 \\ & \\ & \# 601\end{array}$

G CR RG

Snake River

Yellowstone

G CR RG

Snake River

Yellowstone

G CR RG

Snake River

Yellowstone

G CR RG

Snake River

Yellowstone

G CR RG

Snake River

Yellowstone

\#801

\#801

\#801

\#801

G CR RG

Snake River

Yellowstone

\#841

\#841

\#841

\#841

G CR RG

Snake River

Yellowstone
TTCGCACTTC TAATTTATCC TCTTGTTACC ACCCTCACCC TTCGCACTTC TAATTTATCC TCTTGTTACC ACCCTCACCC TTCGCACTTC TAATTTATCC TCTTGTTACC ACCCTCACCC

TTCGCACTTC TAATTTATCC TCTTGTTACC ACCCTCACCC

CAACCCCCCA GCACAAAGAC TGAGCCCTCA CTCACGTAAA CAACCCCCCA GCACAAAGAC TGAGCCCTCA CTCACGTAAA CAACCCCCCA GCACAAAGAC TGAGCCCTCA CTCACGTAAA

CAACCCCCCA GCACAAAGAC TGAGCCCTCA CTCACGTAAA

AACTGCTATC AAAATGGCCT TCCTAGTGAG CTTACTCCCC AACTGCTATC AAAATGGCCT TCCTAGTGAG CTTACTCCCC AACTGCTATC AAAATGGCCT TCCTAGTGAG CTTACTCCCC

AACTGCTATC AAAATGGCCT TCCTAGTGAG CTTACTCCCC

CTTTTTATAT TCCTAGACCA AGGAACCGAA ACTATCGTCA CTTTTTATAT TCCTAGACCA AGGAACCGAA ACTATCGTCA CTTTTTATAT TCCTAGACCA AGGAACCGAA ACTATCGTCA

CTTTTTATAT TCCTAGACCA AGGAACCGAA ACTATCGTCA

CTAATTGACA GTGAATAAAC ACCACAACCT TTGACATTAA CTAATTGACA GTGAATAAAC ACCACAACCT TTGACATTAA CTAATTGACA GTGAATAAAC ACCACAACCT TTGACATTAA

CTAATTGACA GTGAATAAAC ACCACAACCT TTGACATTAA

CCTTAGCTTT AAATTTGACC ACTACTCCGT TATTTTCACC CCTTAGCTTT AAATTTGACC ACTACTCCAT TATTTTCACC CCTTAGCTTT AAATTTGACC ACTACTCCAT TATTTTCACC

CCTTAGCTTT AAATTTGACC ACTACTCCAT TATTTTCACC $*$

CCTATTGCCC TGTACGTAAC CTGATCTATT CTAGAATTCG CCTATTGCCC TGTACGTAAC CTGATCTATT CTAGAATTCG CCTATTGCCC TGTACGTAAC CTGATCTATT CTAGAATTCG

CCTATTGCCC TGTACGTAAC CTGATCTATT CTAGAATTCG

CATCCTGGTA TATACATGCT GACCCCAACA TAAACCGGTT CATCCTGGTA TATACATGCT GACCCCAACA TAAACCGGTT CATCCTGGTA TATACATGCT GACCCCAACA TAAACCGGTT

CATCCTGGTA TATACATGCT GACCCCAACA TAAACCGGTT 


\begin{tabular}{|c|c|c|}
\hline G CR RG & \#921 & CTTTAAGTAC CTCCTCCTCT TCCTGATTGC CATAATTATT \\
\hline Snake River & \#921 & CTTTAAGTAC CTCCTCCTCT TCCTGATTGC CATAATTATT \\
\hline Yellowstone & \#921 & CTTTAAGTAC CTCCTCCTCT TCCTGATTGC CATAATTATT \\
\hline & \#921 & CTTTAAGTAC CTCCTCCTCT TCCTGATTGC CATAATTATT \\
\hline G CR RG & \#961 & TTGGTAACCG CCAACAACAT GTTCCAACTG TTTATCGGCT \\
\hline Snake River & \#961 & TTGGTAACCG CCAACAACAT GTTCCAACTG TTTATCGGCT \\
\hline Yellowstone & \#961 & TTGGTAACCG CCAACAACAT GTTCCAACTG TTTATCGGCT \\
\hline & \#961 & TTGGTAACCG CCAACAACAT GTTCCAACTG TTTATCGGCT \\
\hline G CR RG & $\# 1001$ & GAGAGGGAGT TGGAATTATA TCGTTCCTCC TTATCGGGTG \\
\hline Snake River & \#1001 & GAGAAGGAGT TGGAATTATA TCATTCCTCC TTATCGGGTG \\
\hline Yellowstone & \#1001 & GAGAAGGAGT TGGAATTATA TCATTCCTCC TTATCGGGTG \\
\hline & \#1001 & $\begin{array}{l}\text { GAGAAGGAGT TGGAATTATA TCATTCCTCC TTATCGGGTG } \\
* *\end{array}$ \\
\hline G CR RG & \#1041 & GTGGCACGGT CGAGCCGACG CTAACACAG \\
\hline Snake River & \#1041 & GTGGCACGGT CGAGCCGACG CTAACACAG \\
\hline Yellowstone & \#1041 & GTGGCACGGT CGAGCCGACG CTAACACAG \\
\hline & \#1041 & CTAACACAG \\
\hline
\end{tabular}

